\title{
Outcomes of dose-adjusted Berlin-Frankfurt-Münster-90 regimen without radiotherapy in adolescents and adults with $T$ cell lymphoblastic lymphoma
}

\author{
Yan Xie $^{1} \cdot$ Yuntao Zhang ${ }^{1} \cdot$ Wen Zheng ${ }^{1} \cdot$ Xiaopei Wang ${ }^{1} \cdot$ Ningjing Lin $^{1} \cdot$ \\ Meifeng Tu ${ }^{1} \cdot$ Lingyan Ping ${ }^{1}$ - Zhitao Ying ${ }^{1}$ C Chen Zhang ${ }^{1}$ Weiping Liu ${ }^{1}$. \\ Lijuan Deng ${ }^{1} \cdot$ Yuqin Song ${ }^{1} \cdot$ Jun Zhu ${ }^{1}$
}

Received: 31 December 2014/ Accepted: 26 February 2015/Published online: 10 March 2015

(C) The Author(s) 2015. This article is published with open access at Springerlink.com

\begin{abstract}
The aim of this study was to evaluate the outcomes using the dose-adjusted Berlin-Frankfurt-Munster (BFM-90) regimen without radiotherapy in adolescents and adults with $\mathrm{T}$ cell lymphoblastic lymphoma (T-LBL) at Beijing Cancer Hospital. Between March 2004 and December 2013, 57 newly diagnosed T-LBL patients were treated in our center. We retrospectively analyzed their main clinical characteristics and prognosis. The media age of the patients at diagnosis was 26 (range 14-54). At a median follow-up of 24 months (range 5-119), 38 patients $(67 \%)$ were alive. The estimated 3-year overall survival (OS) rate and progression-free survival (PFS) rate were 64 and $60 \%$, respectively. Abnormal WBC at diagnosis, high IPI and no early response were indicated as adverse prognostic factors for both PFS and OS $(p<0.05)$. There was also a trend for better survival in autologous peripheral blood stem cell transplantation (APBSCT) group as compared to non-APBSCT group (3-year OS 83 vs. $57 \%$ ), but without any significant difference. This study suggested that the dose-adjusted BFM-90 protocol without irradiation showed comparable long-term results in Chinese adolescents and adults with T-LBL. APBSCT may become a choice whether we can identify the best candidate.
\end{abstract}

Keywords T cell lymphoblastic lymphoma . Adolescents · Adults · Dose-adjusted BFM-90 regimen . Prognosis

Jun Zhu

lymphoma6596@163.com

1 Key Laboratory of Carcinogenesis and Translational Research (Ministry of Education), Department of Lymphoma, Peking University Cancer Hospital \& Institute, Beijing, People's Republic of China

\section{Introduction}

$\mathrm{T}$ cell lymphoblastic lymphoma (T-LBL) and $\mathrm{T}$ cell acute lymphoblastic leukemia (T-ALL) are categorized as precursor $\mathrm{T}$ cell malignancy, frequently accompanied by a mediastinal mass and a high prevalence of central nervous system (CNS) involvement [1]. Because T-LBL cell marker expression overlaps that of T-ALL, the clinical distinction between the two entities is arbitrarily determined by the degree of bone marrow (BM) involvement: Patients with more than $25 \%$ lymphoblasts are classified as having T-ALL, whereas those with a lesser degree of marrow replacement are classified as having T-LBL [2]. T cell lymphoblastic lymphoma/leukemia accounts for approximately $3.4 \%$ of all non-Hodgkin lymphomas (NHLs) in China [3]. The conventional CHOP-like regimen produced low complete remission $(\mathrm{CR})$ rate $(17 \%)$ and short media overall survival (OS) (8.5 months) [4]. But the prognosis of LBL has dramatically improved with the use of intensive ALL-type chemotherapy regimens, with an event-free survival (EFS) of $90 \%$ in children and disease-free survival (DFS) of $72 \%$ in adults $[5,6]$. Although the use of ALL-type regimens such as BFM-90 regimen usually acquired better survival rates, they brought more adverse events as well as treatment-related death. The aim of this study was to evaluate the outcomes using the dose-adjusted BerlinFrankfurt-Munster (BFM-90) regimen without radiotherapy in adolescents and adults in our cancer center.

\section{Materials and methods}

\section{Patients}

Between March 2004 and December 2013, patients not less than 14 years old with untreated T-LBL were 
retrospectively analyzed. All cases were pathologically diagnosed by biopsy material from a lymph node or tumor mass according to WHO classification of hematological malignancies in Beijing Cancer Hospital. A series of markers, including CD1a, CD3, CD4, CD8, CD10, CD20, CD45RO, CD79a, Ki-67 and terminal deoxynucleotidyl transferase (TdT), were performed by immunohistochemistry.

A complete panel was performed at the time of baseline staging, re-staging and final evaluation. This panel included a full clinical history, physical examination, complete hematological and biochemical tests, computed tomography (CT) scans of neck, chest, abdomen and pelvic, or $18 \mathrm{~F}$-fluorodeoxyglucose positron emission tomography (18 F-FDG-PET) if possible, cerebrospinal fluid examination, BM aspirate and/or biopsy. Staging was carried out according to the Ann Arbor system for NHL. Diagnosis of central nervous system (CNS) disease was made in case of blast cells found in the cerebrospinal fluid (CSF) or cerebral infiltration on cranial CT. It is important to note that patients who had more than $25 \%$ blasts in the $\mathrm{BM}$ but presented as a bulky tumor were also included in this study.

\section{Treatment}

All patients received a dose-adjusted BFM-90 regimen after they or their guardians signed the informed consent. The protocol was derived from BFM-90 regimen using the same major drugs (prednisone, vincristine, asparaginase, cyclophosphamide, cytarabine, daunorubicin, doxorubicin, methotrexate and 6-mercaptopurine), but the doses and frequency of methotrexate and cytarabine were reduced. Cranial prophylactic radiotherapy was omitted, but all patients received regular intrathecal chemotherapy. Local irradiation was also omitted in this study. The protocols for induction, consolidation, re-induction and maintenance are listed in Table 1. If the patient achieved complete remission (CR) or partial remission (PR) and agreed to receive high-dose chemotherapy plus autologous peripheral blood stem cell transplantation (APBSCT), it should be given after re-induction phase. After APBSCT, the patient would continue with maintenance therapy.

\section{Response evaluation and statistical analysis}

Response was evaluated at the end of each phase of the protocol according to Cheson criteria. Overall survival (OS) was calculated from the date of diagnosis to the date of death by any cause or the last follow-up in survivors. Progression-free survival (PFS) was measured from the date of diagnosis to the first sign of progression, relapse after response, death from any cause or to the date of last follow-up. Patients who were lost to follow-up were censored at the time of their last follow-up examination. OS and PFS curves were estimated using the Kaplan-Meier method and were compared using the log-rank test. Prognostic factors affecting OS and PFS were assessed by the log-rank test. Statistically significant differences were defined as two-sided $p$ values $<0.05$. All statistical analyses were performed using SPSS 13.0 statistical software (SPSS, Chicago, IL).

\section{Results}

\section{Patient characteristics}

A total of 57 patients were consecutive newly diagnosed as T-LBL and treated with the dose-adjusted BFM-90 regimen in our study. Their characteristics are listed in Table 2. Their age ranged from 14 to 54 years, with a median age of 26 years; $72 \%$ cases were male. The majority of patients presented with stage III or IV disease $(47 / 57,82 \%)$. BM infiltration was detected in 33 patients (58\%), 16 (28\%) of whom had more than $25 \%$ blast cells in the BM. Four (7\%) cases were diagnosed as initial CNS invasion, including two patients with concurrent CNS and BM involvement. Twenty-two (39\%) patients presented with bulky mass $(>7.5 \mathrm{~cm})$, and $23(40 \%)$ patients had LDH levels higher than the institutional upper limit of normal (ULN). White blood cells (WBC) were found to be abnormal in $24(42 \%)$ patients at diagnosis. Thirty-four $(60 \%)$ patients were within international prognostic index (IPI) group 0-1, while 23 were within IPI group 2-4. Thirty-four $(69 \%$ ) patients had a Ki-67 score $\geq 75 \%$ (data unavailable in eight patients).

\section{Therapeutic results and survival}

Forty-three (75\%) patients achieved CR and 11 (19\%) achieved PR after induction phase. Among those who achieved CR, 16 patients proceeded to APBSCT. One patient who only achieved PR also received APBSCT after re-induction phase. Three patients had progression disease after induction treatment. Two of them died at 6 and 8 months, respectively. The other patient received a salvage therapy and was still alive at his last follow-up visit.

At a median follow-up of 24 months (range 5-119) for all patients, 38 patients $(67 \%)$ were alive. The estimated 3-year OS and PFS were $64 \%$ and $60 \%$, respectively (Figs. 1, 2). The 3-year OS and PFS were significantly better in patients with normal WBC counts at diagnosis as compared to those with abnormal WBC counts (79 vs. $44 \%, p=0.033 ; 76$ vs. $31 \%, p=0.031$, respectively) (Figs. 3, 4). The 3-year OS and PFS probability was 
Table 1 Protocols of modified BFM-90

\begin{tabular}{|c|c|c|}
\hline Drugs & Doses & Days \\
\hline \multicolumn{3}{|l|}{ Induction phase 1a } \\
\hline Prednisone (PDN) & $60 \mathrm{mg} / \mathrm{m}^{2}$ oral & $1-28$, then taper over $3 \times 3$ days \\
\hline Vincristine (VCR) & $1.5 \mathrm{mg} / \mathrm{m}^{2}(\max 2 \mathrm{mg}) \mathrm{IV}$ & $8,15,22,29$ \\
\hline Daunorubicin (DNR) & $30 \mathrm{mg} / \mathrm{m}^{2} \mathrm{IV}$ & $8,15,22,29$ \\
\hline L-asparaginase (L-ASP) Or Pegasparaginase & $6,000 \mathrm{IU} / \mathrm{m}^{2} \mathrm{IV}$ Or $2500 \mathrm{IU} / \mathrm{m}^{2} \mathrm{IM}$ & $8-15$ Or 8,22 \\
\hline${ }^{\mathrm{a}}$ IT chemotherapy & & ${ }^{\mathrm{b}} 1,15$ \\
\hline \multicolumn{3}{|l|}{ Induction phase $1 b$} \\
\hline Cyclophosphamide (CTX) & $1000 \mathrm{mg} / \mathrm{m}^{2} \mathrm{IV}$ & 1,15 \\
\hline Cytarabine (Ara-C) & $75 \mathrm{mg} / \mathrm{m}^{2} \mathrm{IV}$ & $3-6,17-20$ \\
\hline 6-Mercaptopurine (6-MP) & $60 \mathrm{mg} / \mathrm{m}^{2}$ oral & $1-28$ \\
\hline${ }^{\mathrm{a}}$ IT chemotherapy & & 1,15 \\
\hline \multicolumn{3}{|l|}{ Consolidation phase } \\
\hline 6-Mercaptopurine (6-MP) & $25 \mathrm{mg} / \mathrm{m}^{2}$ oral & $1-28$ \\
\hline${ }^{\mathrm{c}}$ Methotrexate (MTX) & $3000 \mathrm{mg} / \mathrm{m}^{2} \mathrm{IV}$ & 1,15 \\
\hline${ }^{\mathrm{a}}$ IT chemotherapy & & 1,15 \\
\hline \multicolumn{3}{|l|}{ Reinduction phase $2 \mathrm{a}$} \\
\hline Dexamethasone (DXM) & $9 \mathrm{mg} / \mathrm{m}^{2}$ oral & $1-21$, then taper over $3 \times 3$ days \\
\hline Vincristine (VCR) & $1.5 \mathrm{mg} / \mathrm{m}^{2}(\max 2 \mathrm{mg}) \mathrm{IV}$ & $1,8,15,22$ \\
\hline Daunorubicin (DNR) & $30 \mathrm{mg} / \mathrm{m}^{2} \mathrm{IV}$ & $1,8,15,22$ \\
\hline L-asparaginase (L-ASP) Or Pegaspargase & $6,000 \mathrm{IU} / \mathrm{m}^{2} \mathrm{IV}$ Or $2500 \mathrm{IU} / \mathrm{m}^{2} \mathrm{IM}$ & $1-8$ Or 1,15 \\
\hline${ }^{\mathrm{a}}$ IT chemotherapy & & 1,15 \\
\hline \multicolumn{3}{|l|}{ Reinduction phase $2 b$} \\
\hline Cyclophosphamide (CTX) & $1000 \mathrm{mg} / \mathrm{m}^{2} \mathrm{IV}$ & 1,15 \\
\hline Cytarabine (Ara-C) & $75 \mathrm{mg} / \mathrm{m}^{2} \mathrm{IV}$ & $3-6,17-20$ \\
\hline 6-Mercaptopurine (6-MP) & $60 \mathrm{mg} / \mathrm{m}^{2}$ oral & $1-28$ \\
\hline${ }^{\mathrm{a}}$ IT chemotherapy & & 1,15 \\
\hline \multicolumn{3}{|l|}{ Maintenance phase } \\
\hline Methotrexate (MTX) & $20 \mathrm{mg} / \mathrm{m}^{2}$ oral & Once a week for 12 months \\
\hline 6-Mercaptopurine (6-MP) & $60 \mathrm{mg} / \mathrm{m}^{2}$ oral & Daily for 12 months \\
\hline
\end{tabular}

significantly worse in IPI group 2-4 when compared to IPI group $0-1$ (45 vs. $74 \%, p=0.027 ; 34$ vs. $80 \%$, $p=0.004$, respectively) (Figs. 5, 6). Patients with elevated $\mathrm{LDH}$ at diagnosis were significantly associated with poor 3 -year PFS (40\%, $p=0.023$ ), but there was no statistically difference in the 3 -year OS ( $p=0.154)$. There was a significant difference between 3-year OS and PFS by treatment response. A trend of better survival was shown in APBSCT group as compared to non-APBSCT group (3year OS: 83 vs. 57 \%; 3-year PFS: 75 vs. $54 \%$ ); however, the result did not show significant difference. There was no statistically difference in 3-year OS or PFS according to gender, stage, Ki-67, CNS involvement, BM involvement or bulky mass (Table 2).
Twenty-five patients experienced progression or relapse. Among them, 23 cases progressed or relapsed during the first 2 years and the other two cases relapsed at 36 and 68 months, respectively. Sites of relapse included lymph node $(n=17), \mathrm{BM}(n=6)$ and CNS $(n=2)$. All patients with $\mathrm{BM}$ relapse had $\mathrm{BM}$ involvement at diagnosis, but only one patient with CNS relapse had CNS invasion at diagnosis. All of the patients with BM or CNS relapse died. Seven patients with lymph node relapse were alive at the last visit. Five cases were still in salvage therapy, but only one of them achieved PR. One patient accepted allogeneic stem cell transplant and remained in CR at 52 months of follow-up. One patient only accepted salvage chemotherapy and showed no sign of progression at 96 months. 
Table 2 Patients'

characteristics and prognostic factors for overall survival (OS) and progression-free survival (PFS)

\begin{tabular}{|c|c|c|c|c|c|}
\hline Variable & Number (\%) & 3 -year OS (\%) & $p$ value & 3-year PFS (\%) & $p$ value \\
\hline \multicolumn{6}{|l|}{ Gender } \\
\hline Male & $41(72 \%)$ & 63 & & 58 & \\
\hline Female & $16(28 \%)$ & 69 & 0.965 & 70 & 0.879 \\
\hline \multicolumn{6}{|l|}{ Stage } \\
\hline $\mathrm{I}+\mathrm{II}$ & $10(18 \%)$ & 80 & & 88 & \\
\hline $\mathrm{III}+\mathrm{IV}$ & $47(82 \%)$ & 60 & 0.138 & 53 & 0.085 \\
\hline \multicolumn{6}{|l|}{ Ki-67 } \\
\hline$\geq 75 \%$ & $34(69 \%)$ & 71 & & 67 & \\
\hline$<75 \%$ & $15(31 \%)$ & 60 & 0.283 & 52 & 0.518 \\
\hline \multicolumn{6}{|l|}{ LDH } \\
\hline$>\mathrm{ULN}$ & $23(40 \%)$ & 47 & & 40 & \\
\hline$\leq \mathrm{ULN}$ & $34(60 \%)$ & 76 & 0.154 & 72 & 0.023 \\
\hline \multicolumn{6}{|l|}{ WBC } \\
\hline Normal & $33(58 \%)$ & 79 & & 76 & \\
\hline Abnormal & $24(42 \%)$ & 44 & 0.033 & 37 & 0.031 \\
\hline \multicolumn{6}{|c|}{ CNS involvement } \\
\hline Yes & $4(7 \%)$ & 50 & & 25 & \\
\hline No & $53(93 \%)$ & 67 & 0.351 & 65 & 0.180 \\
\hline \multicolumn{6}{|c|}{ BM involvement } \\
\hline Yes & $33(58 \%)$ & 57 & & 42 & \\
\hline No & $24(42 \%)$ & 72 & 0.130 & 76 & 0.060 \\
\hline \multicolumn{6}{|c|}{ Bulky mass $(>7.5 \mathrm{~cm})$} \\
\hline Yes & $22(39 \%)$ & 65 & & 67 & \\
\hline No & $35(61 \%)$ & 64 & 0.731 & 56 & 0.645 \\
\hline \multicolumn{6}{|l|}{ IPI } \\
\hline $0-1$ & $34(60 \%)$ & 80 & & 80 & \\
\hline $2-4$ & $23(40 \%)$ & 45 & 0.027 & 34 & 0.004 \\
\hline \multicolumn{6}{|l|}{ Response } \\
\hline $\mathrm{CR}$ & $43(75 \%)$ & 74 & & 69 & \\
\hline PR & $11(19 \%)$ & 41 & & 23 & \\
\hline PD & $3(5 \%)$ & 0 & 0.000 & 0 & 0.000 \\
\hline \multicolumn{6}{|l|}{ APBSCT } \\
\hline Yes & $17(30 \%)$ & 83 & & 75 & \\
\hline No & $40(70 \%)$ & 57 & 0.133 & 54 & 0.064 \\
\hline
\end{tabular}

\section{Toxicity}

No death occurred due to toxicity. The most common grade 3 and 4 adverse events were hematologic events, including neutropenia (in $86 \%$ cases), thrombocytopenia (in $56 \%$ ), anemia (in $49 \%$ ) and febrile neutropenia (in $46 \%$ ). Grade 3 aminotransferase elevation was seen in six patients. One patient developed acute renal failure due to high-dose methotrexate, and one patient suffered from acute pancreatitis due to pegylated asparaginase. No other grade 3 or 4 non-hematologic adverse events occurred.

\section{Discussion}

T cell lymphoblastic lymphoma (T-LBL) is an uncommon, aggressive but curable $\mathrm{T}$ cell lymphoma in adult, but more common in younger adults [7]. In our study, the patients showed clinical characteristics as published data: younger age, predominantly male, advanced stage and higher BM involvement.

In our study, we tried the dose-adjusted BFM-90 regimen and observed a CR rate of $75 \%$ and a PR rate of $19 \%$ at the end of induction phase, and the 3-year PFS and OS were 60 and $64 \%$, respectively. Despite the lower CR rate, 


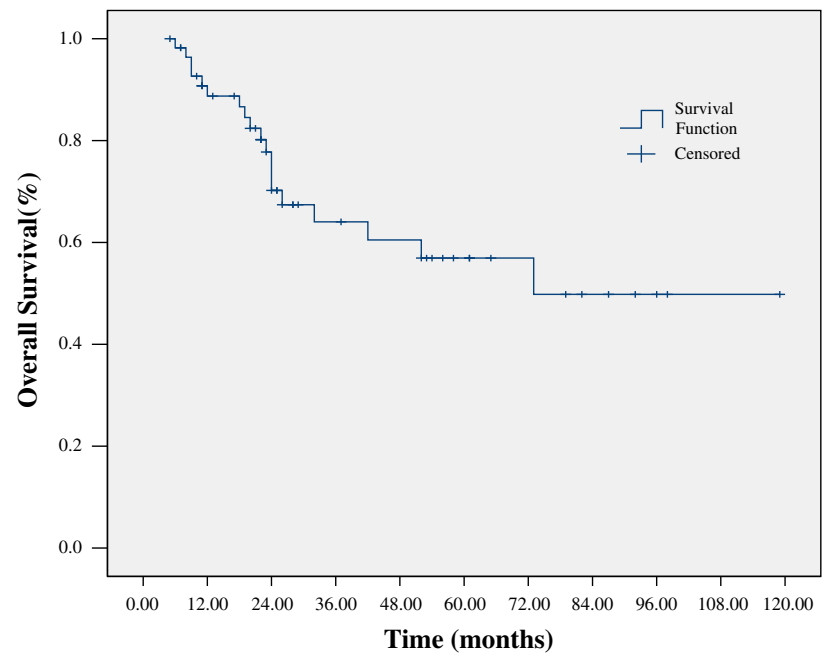

Fig. 1 Overall survival for all patients with T-LBL

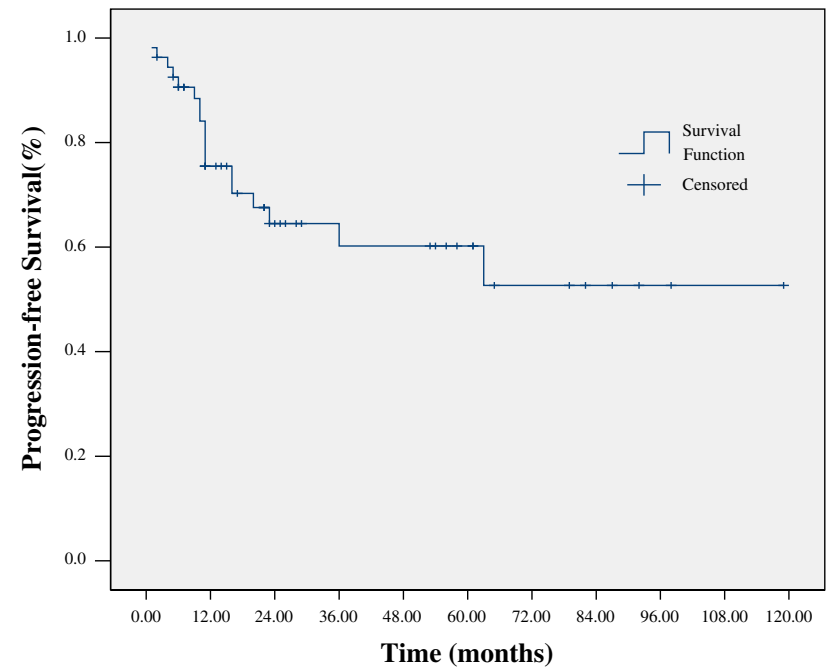

Fig. 2 Progression-free survival for all patients with T-LBL

we achieved a comparable overall response rate (ORR $94 \%$ ) and long-term outcome, comparing favorably with those obtained in Thomas et al. study and Wang $\mathrm{K}$ et al. study $[8,9]$. The most common grade 3 and 4 adverse events still were hematologic events, but overall tolerance was acceptable with no treatment-related mortality. It showed that our dose-adjusted BFM-90 regimen had similar efficacy and acceptable toxicity.

Relapse and progression occurred in 25 of 57 patients in this study, and their outcomes were very poor. If we excluded the five alive patients on therapy, only two patients survived free of the disease in this study, supporting literature about the difficulty in salvaging patients with recurrent T-LBL [10-12]. More effective new drugs such as nelarabine and NOTCH-1 inhibitors are needed to improve the outcome of patients with relapsed disease. Most

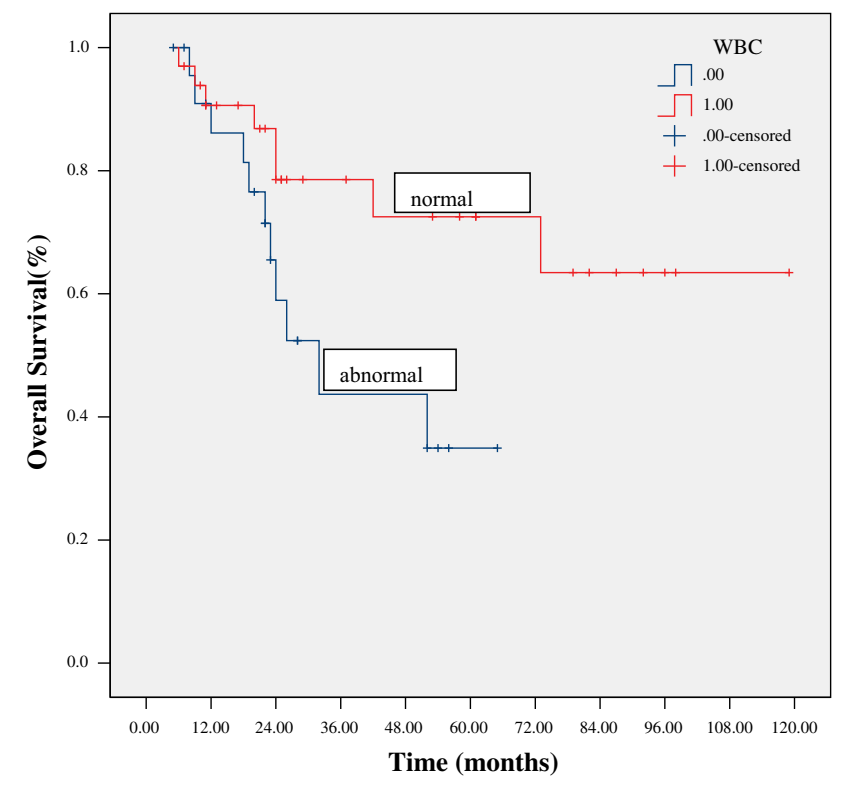

Fig. 3 Overall survival stratified by white blood cell counts at diagnosis

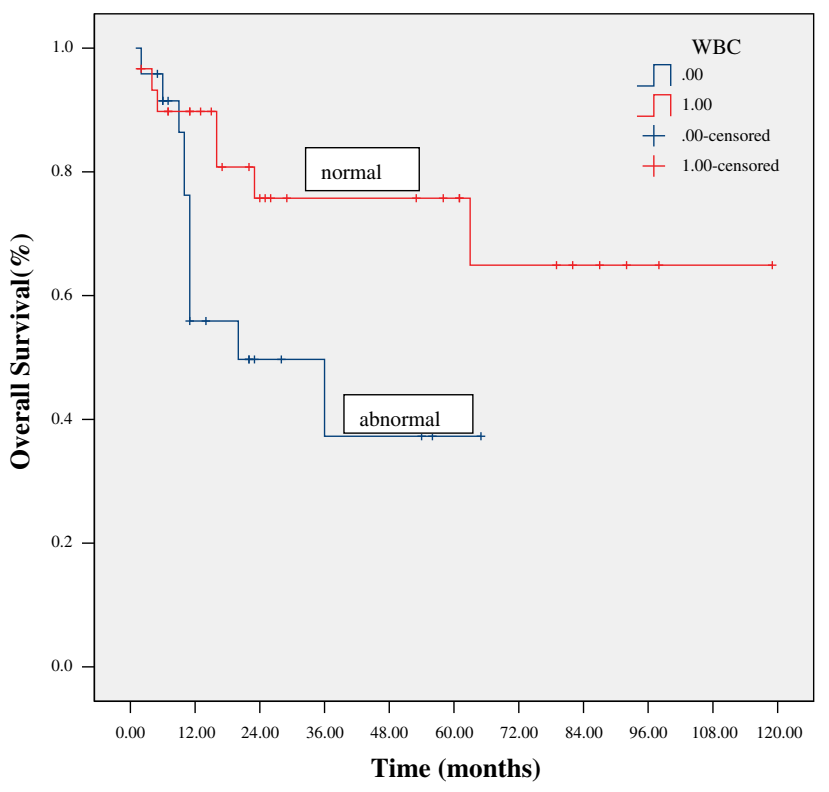

Fig. 4 Progression-free survival stratified by white blood cell counts at diagnosis

relapses occurred within the first 2 years, similar to Termuhlen et al. report [13]. But it should be noted that late relapse still happened up to 68 months. Lymph nodes (17/ 25) remained the most common site of relapse, but with disseminated sites, which suggested that local irradiation might not prevent relapse and systemic therapy was more important. There are no sufficient data to analyze the specific precise values of intrathecal therapy, systemic CNS-penetrating therapy and cranial irradiation as 


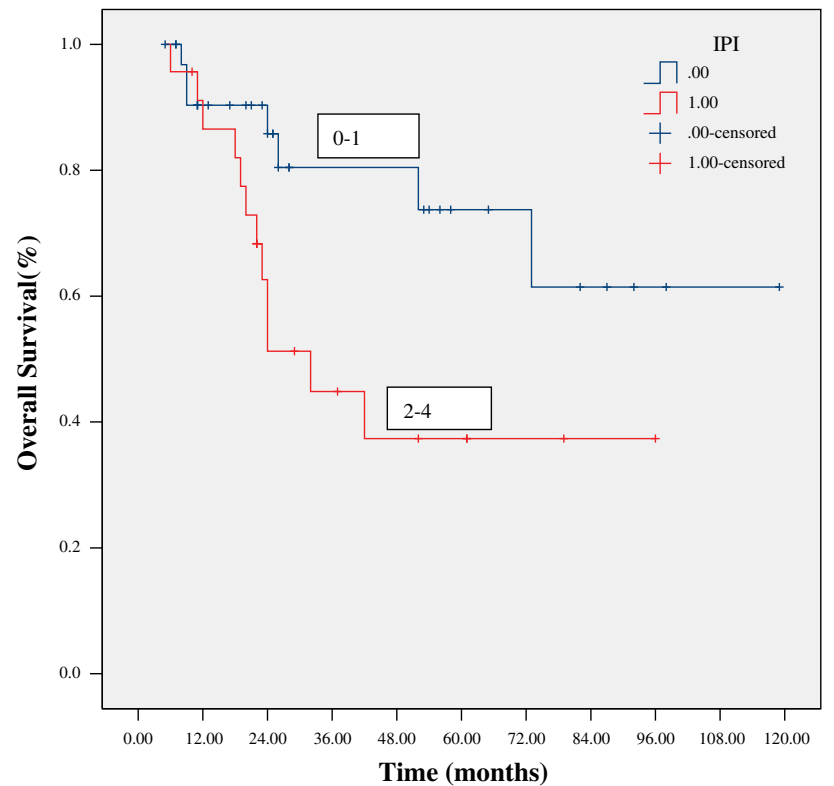

Fig. 5 Overall survival stratified by IPI score at diagnosis

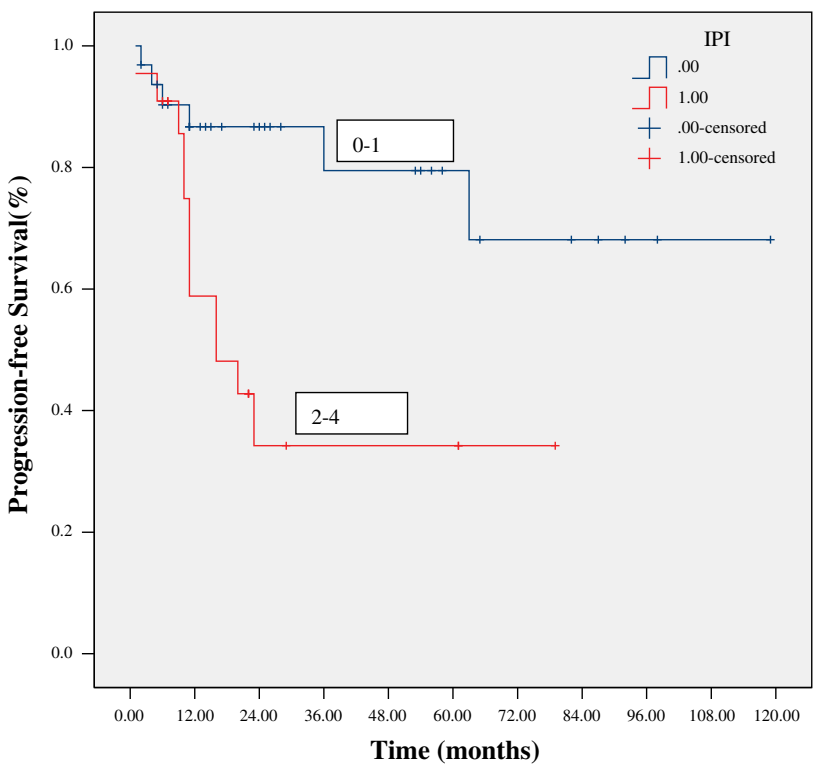

Fig. 6 Progression-free survival stratified by IPI score at diagnosis

prophylaxis methods. Consecutive studies from the BFM group introduced four doses of intravenous methotrexate at $5 \mathrm{~g} / \mathrm{m}^{2}$, and in NHL/BFM-95, prophylactic cranial irradiation was safely eliminated [5, 14]. The Children's Cancer Group (CCG) improved the outcome in ALL patients using a modified BFM approach without high-dose methotrexate or cranial irradiation, and CNS prophylaxis was frequent delivery of intrathecal chemotherapy [13, 15]. In our study, we gave patients two doses of intravenous methotrexate at $3 \mathrm{~g} / \mathrm{m}^{2}$ combined with $10-12$ doses intrathecal chemotherapy without prophylactic cranial irradiation. Two patients $(3.5 \%, 2 / 57)$ had CNS relapse, and one of them had CNS invasion at diagnosis. The incidence of CNS relapse occurred in our group was comparable to that reported by Hoelzer et al. [6] and Möricke et al. [16], all less than $5 \%$. The low incidence of CNS relapse supports the efficacy of such CNS prophylaxis method, but it needs larger scale and prospective trials to confirm.

Song et al. [17] suggested consolidation with SCT in first response in chemosensitive T-LBL adult patients, which showed favorable long-term outcome [17]. Sweetenham et al. [18] conducted a prospective multicenter randomized trial to determine whether autologous stem cell transplantation (ASCT) was superior to conventional-dose consolidation and maintenance chemotherapy (CC) as post-remission therapy in LBL. With a median follow-up of 37 months, the actuarial 3-year relapse-free survival rate was $24 \%$ for the CC arm and $55 \%$ for the ASCT arm $(p=0.065)$. The OS rates were 45 and $56 \%$, respectively $(p=0.71)$. In our study, 17 patients proceeded with APBSCT followed by maintenance therapy. There was also a trend for better survival in APBSCT group compared to non-APBSCT group (3-year OS: 83 vs. $57 \%$ ); however, the result did not show significance difference. We need to increase the number of patients and identify the best candidate for APBSCT.

To date, reliable and reproducible prognostic factors for adult LBL have not been described $[6,9,13,19]$. This study confirms the previously published findings that gender, stage, a bulky mass, elevated LDH, CNS or BM infiltration did not have a significant effect on OS. And in our series, abnormal WBC at diagnosis and high IPI were indicated as adverse prognostic factors for both EFS and OS, in contrast to report from Hoelzer et al. [6]. We also found that early response in such patient was an important prognostic factor. Patients who achieved CR or PR after induction therapy ( 8 weeks) acquired a better outcome. This suggests that we should adjust the treatment strategy as earlier as response evaluation is not satisfactory after induction regimen.

This study suggested that the dose-adjusted BFM-90 protocol without irradiation showed comparable long-term survival and control of CNS recurrence in Chinese adolescents and adults with T-LBL. Although APBSCT in this group did not improve survival, it may become a choice whether we can identify the best candidate.

Conflict of interest The authors declare that they have no conflict of interest.

Ethical standard All procedures performed in studies involving human participants were in accordance with the ethical standards of the institutional and/or national research committee and with the 1964 Helsinki declaration and its later amendments or comparable ethical standards. For this type of study, formal consent is not required. 
Open Access This article is distributed under the terms of the Creative Commons Attribution License which permits any use, distribution, and reproduction in any medium, provided the original author(s) and the source are credited.

\section{References}

1. Swerdlow SH, Campo E, Harris NL, et al. World Health Organization classification of tumours of haematopoietic and lymphoid tissues. Lyon: IARC Press; 2008.

2. Murphy SB. Classification, staging and end results of treatment of childhood non-Hodgkin's lymphomas: dissimilarities from lymphomas in adults. Semin Oncol. 1980;7:332-9.

3. Sun J, Yang Q, Lu Z, et al. Distribution of lymphoid neoplasms in China: analysis of 4,638 cases according to the World Health Organization classification. Am J Clin Pathol. 2012;138(3): 429-34.

4. Chen YC, Ho CL, Kao WY, et al. Adult lymphoblastic lymphoma in Taiwan: an analysis of treatment results of 26 patients. Ann Hematol. 2001;80(11):647-52.

5. Reiter A, Schrappe M, Ludwig WD, et al. Intensive ALL-type therapy without local radiotherapy provides a $90 \%$ event-free survival for children with T-cell lymphoblastic lymphoma: a BFM group report. Blood. 2000;95(2):416-21.

6. Hoelzer D, Gokbuget N, Digel W, et al. Outcome of adult patients with T-lymphoblastic lymphoma treated according to protocols for acute lymphoblastic leukemia. Blood. 2002;99(12): 4379-85.

7. Cortelazzo S, Ponzoni M, Ferreri AJ, et al. Lymphoblastic lymphoma. Crit Rev Oncol Hematol. 2011;79(3):330-43.

8. Thomas DA, O'Brien S, Cortes J, et al. Outcome with the hyperCVAD regimens in lymphoblastic lymphoma. Blood. 2004;104(6): 1624-30.

9. Wang K, Chen X, Wuxiao Z, et al. Long-term outcomes of modified Berlin-Frankfurt-Münster-90 regimen in adults with T-lymphoblastic lymphoma: a single-center experience. Leuk Lymphoma, 2014; Early Online: 1-6.

10. Burkhardt B, Reiter A, Landmann E, et al. Poor outcome for children and adolescents with progressive disease or relapse of lymphoblastic lymphoma: a report from the Berlin-FrankfurtMuenster group. J Clin Oncol. 2009;27(20):3363-9.
11. Cortelazzo S, Intermesoli T, Oldani E, et al. Results of a lymphoblastic leukemia-like chemotherapy program with riskadapted mediastinal irradiation and stem cell transplantation for adult patients with lymphoblastic lymphoma. Ann Hematol. 2012;91:73-82.

12. Abromowitch M, Sposto R, Perkins S, et al. Shortened intensified multi-agent chemotherapy and non-cross resistant maintenance therapy for advanced lymphoblastic lymphoma in children and adolescents: report from the Children's Oncology Group. Br J Haematol. 2008;143(2):261-7.

13. Termuhlen AM, Smith LM, Perkins SL, et al. Disseminated lymphoblastic lymphoma in children and adolescents: results of the COG A5971 trial: a report from the Children's Oncology Group. Br J Haematol. 2013;162(6):792-801.

14. Burkhardt B, Woessmann W, Zimmermann M, et al. Impact of cranial radiotherapy on central nervous system prophylaxis in children and adolescents with central nervous system-negative stage III or IV lymphoblastic lymphoma. J Clin Oncol. 2006;24(3): 491-9.

15. Nachman J, Sather HN, Cherlow JM, et al. Response of children with high-risk acute lymphoblastic leukemia treated with and without cranial irradiation: a report from the Children's Cancer Group. J Clin Oncol. 1998;16(3):920-30.

16. Möricke A, Reiter A, Zimmermann M, et al. Risk-adjusted therapy of acute lymphoblastic leukemia can decrease treatment burden and improve survival: treatment results of 2169 unselected pediatric and adolescent patients enrolled in the trial ALL-BFM 95. Blood. 2008;111(9):4477-89.

17. Song KW, Barnett MJ, Gascoyne RD, et al. Primary therapy for adults with T-cell lymphoblastic lymphoma with hematopoietic stem-cell transplantation results in favorable outcomes. Ann Oncol. 2007;18(3):535-40.

18. Sweetenham JW, Santini G, Qian W, et al. High-dose therapy and autologous stem-cell transplantation versus conventional-dose consolidation/maintenance therapy as postremission therapy for adult patients with lymphoblastic lymphoma: results of a randomized trial of the European Group for Blood and Marrow Transplantation and the United Kingdom Lymphoma Group. J Clin Oncol. 2001;19(11):2927-36.

19. Bonn BR, Rohde M, Zimmermann M, et al. Incidence and prognostic relevance of genetic variations in T-cell lymphoblastic lymphoma in childhood and adolescence. Blood. 2013;121(16): 3153-60. 